\title{
Reduction of skin damage from transcutaneous oxygen electrodes using a spray on dressing
}

\author{
N J EVANS AND N RUTTER \\ Department of Neonatal Medicine and Surgery, City Hospital, Nottingham
}

SUMMARY A spray on, copolymer acrylic dressing (Op-Site) was used to limit the skin damage caused by a transcutaneous oxygen electrode and its adhesive ring. Two identical electrodes were applied to the abdominal skin of 10 preterm infants, one on untreated skin, the other after application of Op-Site. It was found that Op-Site prevented the epidermal damage (as measured by transepidermal water loss) that occurs when the adhesive ring is removed from untreated skin. It did not interfere with transcutaneous oxygen measurements; absolute values and response times were unchanged. Op-Site is therefore useful in preventing the skin trauma that occurs when transcutaneous oxygen monitoring is being performed in preterm infants below 30 weeks' gestation in the first week of life. Care must be taken, however, to prevent a build up of Op-Site-it should be applied as a single layer, allowed to dry, and removed after use.

Skin surface probes are widely used to monitor heart rate, respiratory rate, temperature, and blood gases in sick preterm infants. Such monitoring, though, is not strictly non-invasive. All surface probes need to be secured to the skin with adhesive tape, which strips off the superficial layer of the stratum corneum when it is removed. Although this is of little importance in a term infant, it is of tremendous importance in the very preterm infant below 30 weeks' gestation, whose stratum corneum is thin and poorly developed. Transepidermal water loss increases, absorption of topically applied chemicals occurs more readily, and the stripped area may provide a site for the entry of micro-organisms. ${ }^{1}$

Heated transcutaneous gas electrodes cause the most damage to the preterm infant's skin. The skin in contact with the heated electrode suffers the changes of an early burn, the surrounding skin is damaged by stripping when the adhesive ring is removed, and as the electrode site is usually changed every four hours a large area of skin is subjected to trauma. The neonatal unit at King's College Hospital, London, have used a spray on dressing, Op-Site Spray Dressing (Smith and Nephew, United Kingdom), to limit the skin damage caused by transcutaneous oxygen monitoring. Op-Site is an acrylic copolymer in an ethyl acetate and acetone solvent, which forms a flexible film when sprayed on to the skin. This film is permeable to water vapour and gases.
The aim of this study was to see whether Op-Site protects the preterm infant's skin from damage by the probe and to examine whether or not it interferes with transcutaneous oxygen monitoring.

\section{Subjects}

Ten preterm infants were studied. Their gestation ranged from 26 to 31 weeks (mean (SD) 28.4 (1.8)), their birth weight from 950 to $1560 \mathrm{~g}$ (mean (SD) $1 \cdot 2$ $(0 \cdot 26)$ ), and their age from 1 to 7 days (mean 3.8). All infants were clinically stable and were studied with their mothers' permission. The study was approved by the hospital's ethical committee.

\section{Methods}

Investigation of monitor function. Two identical transcutaneous oxygen electrodes (Radiometer TCM2, Copenhagen, Denmark) were calibrated in air at $43^{\circ} \mathrm{C}$. They were placed on undamaged skin on either side of the upper abdomen of preterm infants-one on untreated skin, the other on skin sprayed for one to two seconds with a layer of Op-Site, which was allowed to dry before application of the electrode. Fifteen minutes after application the electrode readings were compared. Readings and response times were then compared when the infant's inspired oxygen concentration was increased by $20 \%$ and then reduced to the original 
concentration. Response times were measured as the time taken for the transcutaneous oxygen reading to restabilise after the inspired oxygen concentration had been changed. The electrodes were left in place for four hours while continuous transcutaneous oxygen recordings were made.

Investigation of skin damage. Transepidermal water loss in $\mathrm{g} / \mathrm{m}^{2} / \mathrm{h}$ was measured at both skin sites before the electrodes were applied using an Evaporimeter (Ep 1, Servomed, Sweden) as previously described. ${ }^{2}$ Transepidermal water loss was then measured from both sites 30 minutes, four hours, and 12-16 hours after removal of the electrodes. Two measurements of transepidermal water loss were made after removal of the electrodes, one from the central area of skin in contact with the heated electrode and one from the surrounding skin in contact with the adhesive ring used to fix the electrode in place.

Analysis. Statistical analysis was by paired Student's $t$ test and by Mann-Whitney $U$ test.

\section{Results}

Transcutaneous oxygen readings were similar from both electrodes and showed no significant difference, and the response times of the two electrodes were identical (Fig. 1). Two factors, however, did

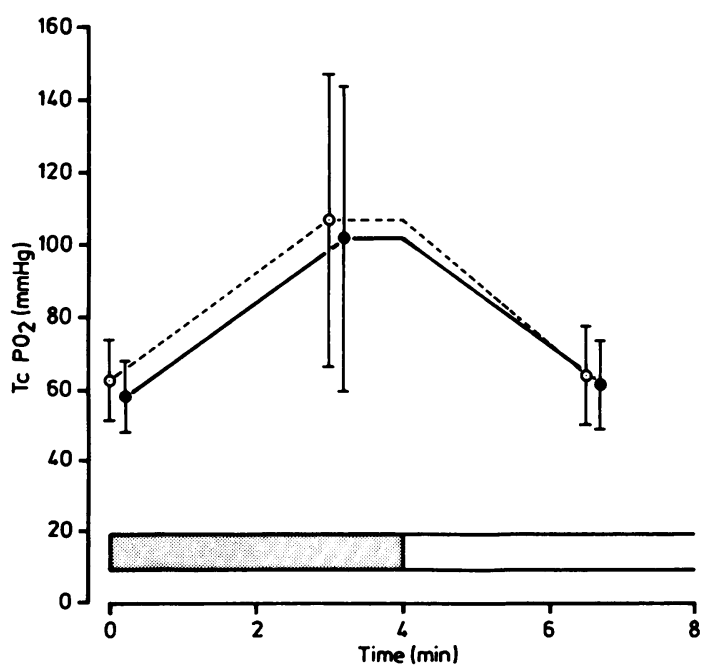

Fig. 1 Mean (SD) transcutaneous oxygen tensions $\left(\mathrm{TcPO}_{2}\right)(\mathrm{mm} \mathrm{Hg})$ from skin treated with $\mathrm{Op}$-Site (O) and untreated skin (O) when the inspired oxygen concentration was increased (shaded area) and then decreased (blank area). affect the transcutaneous oxygen readings. Transcutaneous oxygen readings were lower from skin that had had several layers of Op-Site applied than from untreated skin. In one of the infants studied the transcutaneous oxygen tension from the treated skin after four Op-Site applications was about 10 $\mathrm{mm} \mathrm{Hg}$ lower than at the control site and about 20

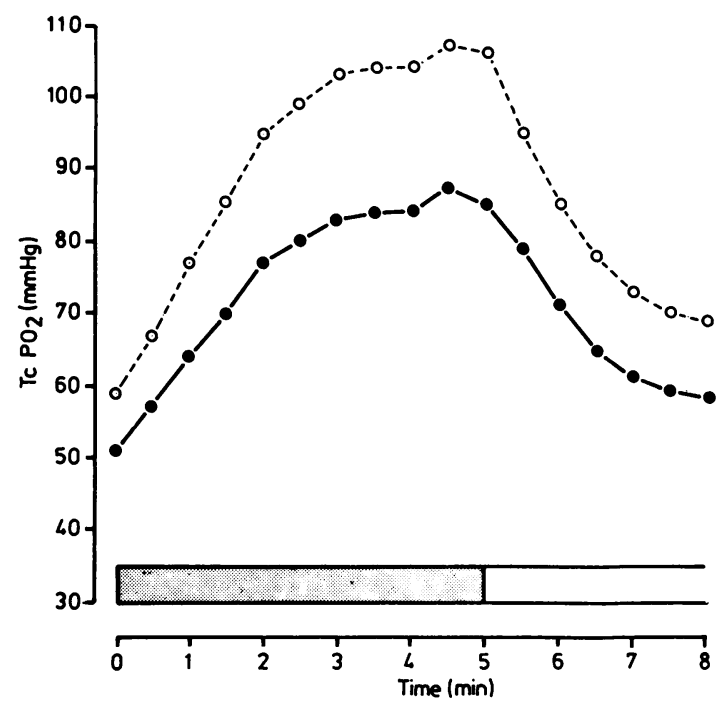

Fig. 2 Transcutaneous oxygen tensions $\left(\mathrm{TcPO}_{2}\right)(\mathrm{mm} \mathrm{Hg})$ recorded from one infant when the inspired concentration was increased (shaded area) and decreased (blank area). Readings from the skin treated with four layers of Op-Site (O) were lower than from untreated skin (O).

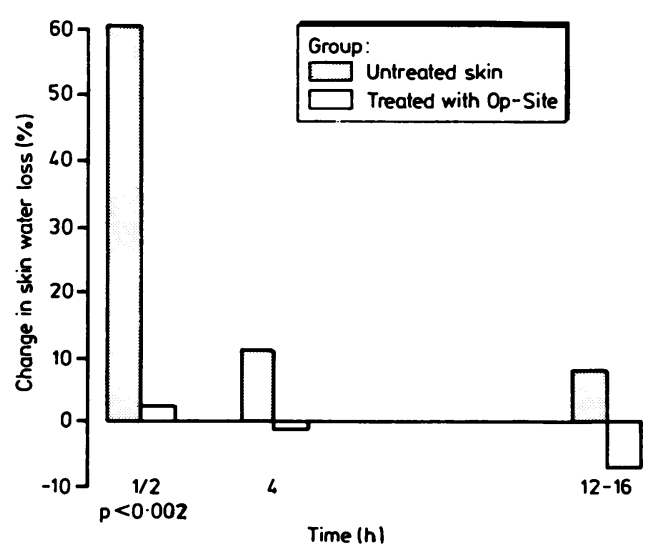

Fig. 3 Change in water loss (\%) from the central area of skin in contact with the heated electrode. The initial high water loss in the untreated group was the result of hydration of the stratum corneum by the contact solution and not the result of epidermal damage. 


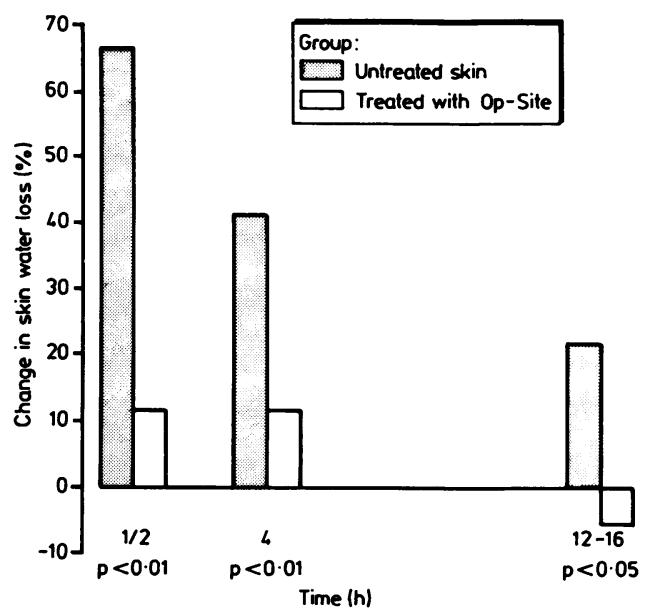

Fig. 4 Change in water loss (\%) from the stripped area of skin in contact with the adhesive ring. The persistently high water loss in the untreated group was the result of damage to the stratum corneum.

$\mathrm{mm} \mathrm{Hg}$ lower when the infant's inspired oxygen concentration was increased (Fig. 2). It was also discovered that if the Op-Site was not allowed to dry before the electrode was applied the solvent affected the oxygen permeability of the electrode membrane, giving falsely high transcutaneous oxygen values.

The percentage change in transepidermal water loss after removal of the electrode was calculated by comparison with the transepidermal water loss before the electrode was applied. Transepidermal water loss was high from the central area of skin 30 minutes after removal of the electrode from the untreated site but not from the site treated with OpSite (Fig. 3), but by four hours the difference was small and not significant. Transepidermal water loss from the skin in contact with the adhesive ring was significantly higher from the untreated skin compared with the skin treated with Op-Site where the rise in transepidermal water loss was small. This difference was still present $12-16$ hours later (Fig. 4).

There was no difference in the disappearance of the central erythema caused by the heated electrode at the treated and untreated sites. Only one mild first degree burn was seen, and this was on untreated skin.

\section{Discussion}

Op-Site spray dressing, when thinly applied, does not interfere with the normal functioning of a transcutaneous oxygen electrode, suggesting that the film is readily permeable to oxygen. This is consistent with previous unpublished observations where we have shown that the thin copolymer layer is permeable to water vapour, carbon dioxide, and oxygen. In the two studies in which more than one layer of Op-Site was applied, however, transcutaneous oxygen measurements were artificially low (as in Fig. 2).

We were unable to show a protective effect of Op-Site on that part of the skin that was in direct contact with the heated electrode.

The transiently high transepidermal water loss seen in the untreated skin after removal of the electrode is probably due to loss of water from the stratum corneum, which has been hydrated by the contact solution. This rapid rate of fall in transepidermal water loss is seen when other water containing preparations are applied to the skin ${ }^{3}$ and when the stratum corneum is drying out in the first few hours after delivery. ${ }^{4}$ Erythema of the skin was mild and transient in both groups, with only one minor burn occurring. We noted, though, that Op-Site abolishes the sensation of heat when the heated electrode is applied to adult skin and it might be expected to reduce the incidence of burns that are occasionally seen with use of the electrode in preterm infants. ${ }^{56}$

Op-Site has an appreciable protective effect on the skin that is in contact with the adhesive ring used to hold the electrode in place. The persistent increase in transepidermal water loss seen in untreated skin is an indication of damage to the stratum corneum caused by stripping. No such damage occurs with the use of Op-Site. Damage to the stratum corneum from stripping is only important in infants below 30 weeks' gestation in the early neonatal period as this is the time when the epidermal barrier is thin, poorly keratinised, ${ }^{7}$ and readily permeable to water vapour and chemicals. ${ }^{1}$ It has been estimated that in such infants, who often have several monitoring probes applied to their skin at any given time, $15 \%$ of the body's skin can be traumatised by adhesive stripping each day ${ }^{1}$ (assuming that transcutaneous gas electrodes are moved every four hours). Besides increasing difficulties in fluid balance and temperature control and the increased risk of poisoning from topically applied agents, secondary skin sepsis may occasionally occur and it is possible that bacteria can enter the body at these sites.

Is it safe to apply Op-Site to the skin of very preterm infants? We observed no local or systemic effects, and it is unlikely that the high molecular weight acrylic copolymer could be absorbed. The solvent quickly evaporates after application of the spray dressing, too quickly for absorption to take 
place. Our study shows that Op-Site protects the skin from adhesive stripping without interfering with transcutaneous oxygen monitoring and could therefore be used with benefit in the most vulnerable infants (below 30 weeks' gestation and less than 1 week old). The finding that underreading of transcutaneous oxygen values occurs if several layers of Op-Site are interposed between electrode and skin, however, is potentially dangerous. To avoid this, the following procedure may be followed. The can of Op-Site is shaken and then held inverted over the skin at a distance of about $10 \mathrm{~cm}$. A one to two second spray is applied to a circle of skin slightly larger than the adhesive electrode ring, using a cardboard or plastic template with a suitable hole cut in it: this limits the Op-Site to the desired area. It must then be allowed to dry for three or four minutes before the electrode is applied. When the electrode is removed the copolymer dressing under the adhesive ring will be removed with it. Any surplus Op-Site can be removed with an isopropyl swab. If Op-Site is repeatedly applied and not removed several layers of copolymer may be built up, which can cause serious underestimation of transcutaneous oxygen tensions.
We are grateful to Dr D Curnock and Professor A Milner for allowing us to study infants under their care and to V A Howe Ltd (London) for lending us the transcutaneous oxygen monitor. $\mathbf{N ~ J}$ Evans was supported by a grant from the Medical Research Council of Great Britain. We are grateful to Dr Harold Gamsu, Consultant Neonatologist at King's College Hospital, London, who suggested that the use of a protective spray on dressing should be examined.

\section{References}

${ }^{1}$ Harpin VA, Rutter N. Barrier properties of the newborn infant's skin. J Pediatr 1983;100:614-9.

2 Nilsson GE. Measurement of water exchange through the skin. Med Biol Eng Comput 1977;15:209-18.

3 Rutter N, Hull D. Water loss from the skin of term and preterm infants. Arch Dis Child 1979;54:858-68.

${ }^{4}$ Rutter N, Hull D. Reduction of skin water loss in the newborn. I. Effect of applying topical agents. Arch Dis Child 1981;56: 669-72.

${ }^{5}$ Boyle RJ, Oh W. Erythema following transcutaneous oxygen monitoring. Pediatrics 1980;65:333-4.

${ }^{6}$ Golden SM. Skin craters-a complication of transcutaneous oxygen monitoring. Pediatrics 1981;67:514-6.

${ }^{7}$ Evans NJ, Rutter N. Development of the epidermis in the newborn. Biol Neonate 1986;49:74-80.

Correspondence to Dr N Rutter, Department of Neonatal Medicine, City Hospital, Hucknall Road, Nottingham NG5 1PB, England.

Received 5 June 1986 